somewhat higher. Small differences in the measurement of the isotopic shifts produce appreciable differences in the partition function ratios and the measurement of the isotopic frequencies with a grating spectrometer would be necessary for greatest precision. As pointed out by Bigeleisen and Friedman and by McCrea, the equi- librium constant for the intramolecular equilibrium

$$
\mathrm{N}^{15} \mathrm{~N}^{14} \mathrm{O}^{16} \rightleftharpoons \mathrm{N}^{14} \mathrm{~N}^{15} \mathrm{O}^{16}
$$

can be calculated by dividing the numbers in column two by those in column one in Table V. This results in an equilibrium constant of 1.0545 at $25^{\circ} \mathrm{C}$.

\title{
Ion Exchange Kinetics. A Nonlinear Diffusion Problem*广
}

\author{
F. Helfferich and M. S. Plesset \\ California Institute of Technology, Pasadena, California
}

(Received October 17, 1957)

\begin{abstract}
Ideal limiting laws are calculated for the kinetics of particle diffusion controlled ion exchange processes involving ions of different mobilities between spherical ion exchanger beads of uniform size and a well-stirred solution. The calculations are based on the nonlinear Nernst-Planck equations of ionic motion, which take into account the effect of the electric forces (diffusion potential) within the system. Numerical results for counter ions of equal valence and six different mobility ratios are presented. They were obtained by use of a digital computer. This approach contains the well-known solution to the corresponding linear problem as a limiting case. An explicit empirical formula approximating the numerical results is given.
\end{abstract}

\section{INTRODUCTION}

$\mathrm{A}^{\mathrm{N}}$ $\mathrm{N}$ ion exchange resin consists essentially of a threedimensional cross-linked network of hydrocarbon chains carrying fixed ionic groups, the electric charge of which is compensated for by mobile ions of opposite charge ("counter ions"). These counter ions are free to diffuse within the resin network. In contact with an electrolyte solution the resin takes up solvent and some additional mobile ions (additional counter ions, and "coions" having the same charge sign as the fixed ionic groups). In an ion exchange process the counter ion species present initially is replaced by another species.

We consider the ion exchange between spherical ion exchange resin beads of uniform size containing the counter ion species $A$ and a well-stirred solution containing the counter ion species $B$. During the process ions $A$ diffuse out of the bead and are replaced by an equivalent amount of ions $B$.

It has been established that such processes are diffusion controlled: the rate determining mechanism is the interdiffusion of the two species $A$ and $B$, either within the resin particles or in a Nernst diffusion layer ("film") adherent to the particle surface, which is not affected by stirring. ${ }^{1}$ Limiting laws for film controlled exchange in ideal systems have been given previously. ${ }^{2}$ In this paper, ideal limiting laws for particle controlled processes are calculated. Particle control is favored by concentrated

* This investigation was supported in part by the Office of Naval Research.

+ Contribution No. 2265 from the Gates and Crellin Laboratories of Chemistry.

${ }^{1}$ Boyd, Adamson, and Myers, J. Am. Chem. Soc. 69, 2836 $(1947)$.

${ }_{2}^{2}$ R. Schlögl and F. Helfferich, J. Chem. Phys. 26, 5 (1957). solutions, large diameter and high degree of crosslinking of the beads, and efficient stirring. ${ }^{3}$

The driving "force" for the flux of an ionic species is, in systems without convection, the gradient of its general chemical potential, the principal elements of which are the concentration gradient of the species and the gradient of the electric potential. Even if no external electric field is applied, a gradient of the electric potential will, as a rule, be built up by the diffusion process (diffusion potential). The corresponding differential equations are nonlinear. The interdiffusion process considered here can be described in terms of one interdiffusion coefficient which, however, is not constant but changes with the concentration ratio of the two counter ion species present. Hence, the mathematical treatment is that of a diffusion problem with varying diffusion coefficient. Hitherto, only the corresponding linear problem, i.e., with constant interdiffusion coefficient, has been solved ${ }^{4}$ and applied to ion exchange kinetics. ${ }^{5-10}$ In our approach the solution to the linear problem represents a limiting case: the exchange of counter ions of equal mobility. The linear solution holds rigorously for isotopic exchange processes, whereas for other cases the deviations are considerable.

${ }^{3}$ F. Helfferich, Angew. Chem. 68, 693 (1956).

${ }^{4}$ R. M. Barrer, Diffusion in and through Solids (Cambridge University Press, London, 1941), p. 29.

${ }^{5}$ Conway, Green, and Reichenberg, Trans. Faraday Soc. 50, 511 (1954).

${ }^{6}$ D. K. Hale and D. Reichenberg, Discussions Faraday Soc. 7, 79 (1949).

${ }_{7}$ T. R. E. Kressman and J. A. Kitchener, Discussions Faraday Soc. 7, 90 (1949).

8 J. B. Rosen, J. Chem. Phys. 20, 387 (1952).

9 D. Reichenberg, J. Am. Chem. Soc. 75, 589 (1953).

${ }^{10}$ E. Glueckauf, Trans. Faraday Soc. 51, 1540 (1955). 


\section{MODEL AND SIMPLIFYING ASSUMPTIONS}

We use the simplest model conceivable and the simplest equations proved to be adequate for the description of ionic diffusion processes, the NernstPlanck equations. ${ }^{11-15}$

Without respect to its actual porous structure the whole resin is treated as a quasi-homogeneous phase. Individual diffusion constants for the mobile ions present are defined correspondingly; they can be measured by tracer techniques in equilibrium systems. ${ }^{1,15-18}$ The assumption is made with experimental support ${ }^{15,16,18}$ that these individual diffusion constants are essentially constant for a given resin, i.e., independent of ionic composition. We make use of the ideal Einstein relation between the individual diffusion constants and the electrochemical mobilities. We neglect any coupling of ionic fluxes other than by electric forces. We assume a constant concentration of fixed ionic groups throughout the resin. Any concentration change and flux of the coions is disregarded, since they are small as compared with those of the counter ions if the concentration of the solution is not too high and that of the fixed ionic groups not too low. ${ }^{13}$ We neglect changes in ionic activity coefficients and swelling condition of the resin as well as the effect of gradients of swelling pressure. Under the simple boundary condition used here, the magnitude of the selectivity coefficient is irrelevant. ${ }^{19}$ We restrict ourselves to radial diffusion, i.e., spherical symmetry. We assume that interdiffusion within the resin beads is the rate determining mechanism throughout the process. This last assumption is not strictly valid; it leads to exchange fluxes that are infinite initially, whereas the possible diffusion rate through the film is finite. For a very short initial period film diffusion must be the rate controlling step.

The assumptions and simplification listed above are those usually made in the formulation of a model for ion exchange kinetics. ${ }^{5-10}$

We do not neglect the effect of the electric potential gradient on the ionic fluxes; in this respect our approach differs from previous theories.

\section{FORMULATION OF THE PROBLEM}

Under the assumptions mentioned, the fluxes of the two counter ion species $A$ and $B$ are given by the

${ }^{11}$ W. Nernst, Z. physik. Chem. (Leipzig) 2, 613 (1888) ; 4, 129 (1889).

${ }_{12}$ M. Planck, Ann. Physik. 39, 161 (1890)

${ }_{13} \mathrm{~T}$. Teorell, Z Elektrochem. 55, 460 (1951).

${ }^{14}$ R. Schlögl, Z. physik. Chem. (Frankfurt) 1, 305 (1954).

1.5 F. Helfferich and H. D. Ocker, Z. physik. Chem. (Frankfurt) 10, 213 (1957).

if B. A. Soldano and G. E. Boyd, J. Am. Chem. Soc. 75, 6107 (1953).

${ }_{17}$ M. Tetenbaum and H. P. Gregor, J. Phys. Chem. 58, 1156 (1954).

${ }^{18}$ B. Stein, Thesis, Göttingen (1957).

${ }_{19}$ F. Helfferich, Discussions Faraday Soc. 21, 83 (1956).
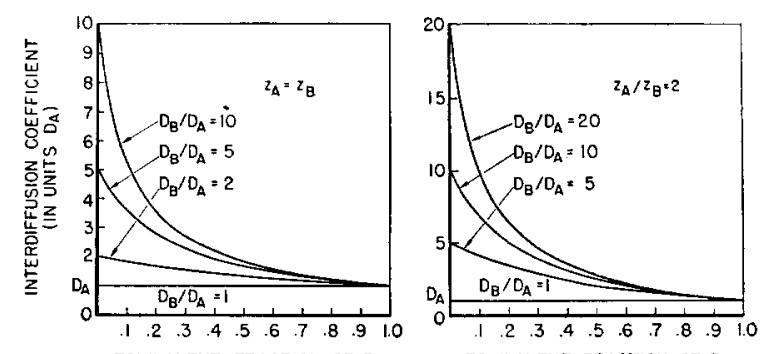

EQUIVALENT FRACTION OF $B$

EQUIVALENT FRACTION OF B

FIG. 1. Dependence of the interdiffusion coefficient $D_{A B}$ on the ionic composition of the exchanger, calculated from Eq. (4), for the interdiffusion of ions of equal valence (left) and of a univalent and a bivalent ion (right). The different curves correspond to different ratios $D_{A} / D_{B}$.

Nernst-Planck equations ${ }^{11-14}$

$$
\begin{gathered}
\boldsymbol{\Phi}_{A}=-D_{A}\left[\operatorname{grad} C_{A}+z_{A} C_{A}(\mathscr{F} / R T) \operatorname{grad} \varphi\right], \\
\boldsymbol{\Phi}_{B}=-D_{B}\left[\operatorname{grad} C_{B}+z_{B} C_{B}(\mathcal{F} / R T) \operatorname{grad} \varphi\right],
\end{gathered}
$$

where $\boldsymbol{\Phi}$ is the flux, $D$ is the individual diffusion constant, $C$ is the molar concentration, $z$ is the electrochemical valence, $\mathcal{F}$ is the Faraday constant, $R$ is the gas constant, $T$ is the absolute temperature, $\varphi$ is the electric potential, and the subscripts $A$ and $B$ refer to the counter ion species.

Electroneutrality requires that the total equivalent concentration of counter ions is constant throughout the bead, since the concentrations of both the fixed ionic groups and the co-ions are constant; thus

$$
z_{A} C_{A}+z_{B} C_{B} \equiv C=\text { const. }
$$

The absence of an electric current in combination with (2) gives the condition

$$
z_{A} \boldsymbol{\Phi}_{A}+z_{B} \boldsymbol{\Phi}_{B}=0 .
$$

By use of (2) and (3), Eqs. (1a) and (1b) may be combined to give

$$
\boldsymbol{\Phi}_{A}=-\left[\frac{D_{A} D_{B}\left(z_{A}{ }^{2} C_{A}+z_{B}{ }^{2} C_{B}\right)}{D_{A} z_{A}{ }^{2} C_{A}+D_{B} z_{B}{ }^{2} C_{B}}\right] \operatorname{grad} C_{A} .
$$

The quantity in brackets may be termed the interdiffusion coefficient $D_{A B}$ of the process. Its value depends not only on the individual diffusion constants $D_{A}$ and $D_{B}$; it is also a function of the concentration ratio $C_{A} / C_{B}$ and therefore a function of the time and space coordinates. The dependence of the interdiffusion coefficient on ionic composition is shown in Fig. 1 for various values of the ratio $D_{A} / D_{B}$. For $C_{A} \ll C_{B}, D_{A B}$ assumes the value $D_{A}$, and for $C_{B} \ll C_{A}$ the value $D_{B}$. The ion present in smaller concentration always has the stronger influence on the interdiffusion coefficient. ${ }^{3,19}$ This simple rule is a consequence of the fact that in (1) the concentration of the species enters in the electric term. It is also physically evident since the electric field acts on every ion present and thus causes a large transference of the species present in high concentration, whereas the 
flux of the species present in low concentration is hardly affected.

For the treatment of time-dependent processes the equation of continuity must be introduced

$$
\partial C_{A} / \partial t=-\operatorname{div} \boldsymbol{\Phi}_{A}
$$

In previous work on time-dependent processes, only the limiting linear case has been considered, to which (4) and (5) reduce when a constant interdiffusion coefficient $D_{A B}$ is assumed. Analytical solutions to the linear problem have been derived for a number of systems including those with spherical symmetry with various boundary conditions. ${ }^{4,20}$ However, Eq. (4) and Fig. 1 show that the assumption of a constant interdiffusion coefficient holds only if the individual diffusion constants $D_{A}$ and $D_{B}$ are equal, as is the case for example in isotopic exchange processes.

This paper deals with the general, nonlinear problem and will treat specifically systems with spherical symmetry. Upon substitution of (4) into (5) an expression is obtained which can be written conveniently in dimensionless form with the introduction of the following dimensionless variables and parameters:

$$
\begin{aligned}
& \gamma \equiv z_{A} C_{A} / C ; \quad \tau \equiv D_{A} t / r ; \quad \rho \equiv r / r_{0} ; \\
& a \equiv z_{A} D_{A} / z_{B} D_{B}-1 ; \quad b \equiv z_{A} / z_{B}-1,
\end{aligned}
$$

where $t$ is time, $r$ is the radial coordinate, and $r_{0}$ is the radius of the beads. The resulting equation

$$
\begin{aligned}
\frac{\partial \gamma}{\partial \tau}-\frac{1}{1+a \gamma}\left[(1+b \gamma) \frac{\partial^{2} \gamma}{\partial \rho^{2}}\right. & \\
& \left.+\frac{b-a}{1+a \gamma}\left(\frac{\partial \gamma}{\partial \rho}\right)^{2}+\frac{2(1+b \gamma)}{\rho} \frac{\partial \gamma}{\partial \rho}\right]=0
\end{aligned}
$$

was derived in a preliminary note. ${ }^{21}$ The fraction $q_{A}$ of the species $A$ still present in the sphere at the dimensionless time $\tau$ is expressed in terms of the solution $\gamma(\rho, \tau)$ of Eq. (7) as follows:

$$
q_{A}(\tau)=3 \int_{0}^{1} \gamma(\rho, \tau) \rho^{2} \partial \rho .
$$

The ion exchange rate, defined as the decrease with time of the amount $Q_{A}$ of the species $A$ (in moles) present in the spheres, may be written as

$$
-\frac{d Q_{A}}{d t}=-V C \frac{d q_{A}}{d t}=-\frac{V C D_{A}}{r_{0}^{2}} \frac{d q_{A}}{d \tau},
$$

where $V$ is the total volume of ion exchanger used.

If the problem is limited to the exchange of counter ions of equal valence $\left(z_{A}=z_{B}\right)$, so that $b=0$, the general

\footnotetext{
${ }^{20}$ S. Patterson, Proc. Phys. Soc. (London) 59, 50 (1947).

21 F. Helfferich and M. S. Plesset, J. chim. phys. (to be published).
}

Eq. (7) may be simplified by the introduction of the new variableł

to become

$$
u \equiv \ln (1+a \gamma)
$$

$$
\frac{\partial u}{\partial \tau}-\frac{e^{-u}}{\rho^{2}} \frac{\partial}{\partial \rho}\left(\rho^{2} \frac{\partial u}{\partial \rho}\right)=0
$$

which is a convenient form for numerical integration. The results reported at the present time will be for this case. Determination of the solutions of Eq. (7) for nonzero values of $b$ are planned for a later time.

We use the simplest initial and boundary conditions possible, assuming that initially the beads contain counter ions $A$ only and the solution counter ions $B$ only, and that no concentration changes occur in the solution.

$$
\begin{array}{lrl}
0 \leqslant \rho<1, & \tau=0, & \gamma(\rho)=1, \\
\rho \geqslant 1, & \gamma(\rho, \tau)=0 .
\end{array}
$$

The boundary condition (12b) corresponds to infinite solution volume or to continuous renewal of the solution. The selection of a boundary condition other than (12b) would require the introduction of further parameters which, for the numerical evaluation we carried out, would have to be chosen arbitrarily and have led to results applicable in special cases only. In contrast, by using the conditions (12), more general limiting laws are obtained, which can be approached fairly closely by experimental techniques. Other initial conditions are treated in Sec. (6).

Even under the simple conditions (12) an analytical solution to the Eqs. (7) or (11) was not obtained. We have evaluated (11) numerically for six values of the ratio $D_{A} / D_{B}$ of the individual diffusion constants :

$$
\begin{array}{ll}
D_{A} / D_{B}=10(a=9), & D_{A} / D_{B}=\frac{1}{10}(a=-0.9), \\
D_{A} / D_{B}=5(a=4), & D_{A} / D_{B}=\frac{1}{5}(a=-0.8), \\
D_{A} / D_{B}=2(a=1), & D_{A} / D_{B}=\frac{1}{2}(a=-0.5) .
\end{array}
$$

The results are given in Sec. (5).

\section{CALCULATION PROCEDURE}

For the numerical evaluation, (11) was approximated by the finite difference form

$$
\begin{aligned}
u(\rho, \tau+\Delta \tau)= & u(\rho, \tau)+g(\rho, \tau) \\
& \times\left\{R_{+}(\rho)[u(\rho+\Delta \rho, \tau)-u(\rho, \tau)]\right. \\
& \left.\quad-R_{-}(\rho)[u(\rho, \tau)-u(\rho-\Delta \rho, \tau)]\right\}
\end{aligned}
$$

where

$$
\begin{aligned}
& g(\rho, \tau) \equiv \exp [-u(\rho, \tau)] \Delta \tau /(\Delta \rho)^{2} ; \\
& R_{+}(\rho) \equiv\left[\left(\rho+\frac{1}{2} \Delta \rho\right) / \rho\right]^{2} ; \\
& R_{-}(\rho) \equiv\left[\left(\rho-\frac{1}{2} \Delta \rho\right) / \rho\right]^{2} .
\end{aligned}
$$

$\ddagger$ It should be noted that the new variable $u$ is related to the electric potential $\varphi$ by $u=-\left(z_{A} \mathfrak{F} / R T\right) \varphi$. 
By use of (13), $u(\rho, \tau+\Delta \tau)$ can be calculated from $u(\rho+\Delta \rho, \tau), u(\rho, \tau)$, and $u(\rho-\Delta \rho, \tau)$. For the calculation the initial condition and the boundary conditions for $\rho=0$ and $\rho=1$ are required. The initial condition is obtained from (12a) and (10):

$$
u(\rho, \tau=0)=\ln (1+a) .
$$

The boundary conditions at the bead surface $(\rho=1)$ is given by (12b) and (10):

$$
u(\rho=1, \tau)=0 .
$$

It can be shown that the gradients of $\gamma$ and $u$ vanish at the center of the sphere:

$$
(\partial u / \partial \rho)_{\rho=0}=0 .
$$

Hence the condition at $\rho=0$ becomes

$$
u(\rho=0, \tau)=u(\rho=0+\Delta \rho, \tau) .
$$

By choice of $\Delta \rho$ an upper limit is given for $\Delta \tau$ by the stability condition for the numerical integration. ${ }^{22}$ In case of (13), this condition is

$$
\Delta \tau \leqslant 0.4 e^{u}(\Delta \rho)^{2} .
$$

The function $u(\rho, \tau)$ was calculated numerically from (13) and (14) in accord with the requirement (15).

For the numerical integration corresponding to (8), which leads to the fraction $q_{A}$ still present in the sphere, Simpson's rule was used:

$$
\begin{aligned}
q_{A}(\tau)= & \frac{3}{a} \int_{0}^{1}\left[e^{u(\rho, \tau)}-1\right] \rho^{2} \partial \rho \\
= & \frac{(\Delta \rho)^{3}}{a}\left\{4 \sum_{n=1,3,5 \ldots}^{1 / \Delta \rho-1} n^{2}\left[e^{u(\rho, \tau)}-1\right]\right. \\
& \left.+2 \sum_{n=2,4,6 \ldots}^{1 / \Delta \rho-2} n^{2}\left[e^{u(\rho, \tau)}-1\right]\right\},
\end{aligned}
$$

where

$$
n \equiv \rho / \Delta \rho .
$$
by

The dimensionless rate, $-d q_{A} / d \tau$, was approximated

$$
-d q_{A} / d \tau=\left[q_{A}(\tau-\Delta \tau)-q_{A}(\tau)\right] / \Delta \tau .
$$

The calculation starts out with a step function. Therefore, a very fine subdivision of space and time is required initially. The initial spacing of $\rho$ used was $\Delta \rho=1 / 640$. The spacing of $\Delta \tau$ was selected in accordance with the stability condition (15). As the calculation proceeded, $\Delta \rho$ was increased stepwise to $1 / 320,1 / 160$, $1 / 80,1 / 40$ (at about $q_{A}=0.9$ ), $1 / 20$ (at about $q_{A}=0.6$ ), and $1 / 10\left(\right.$ at $\left.q_{A}<0.1\right)$. $\Delta \tau$ was increased correspondingly. The estimated maximum error in $q_{A}$ is \pm 0.01 ; the error in $d q_{A} / d \tau$ is about $\pm 2 \%$.

The calculations were carried out on the Datatron digital computer (manufactured by Electrodata, Inc.,

\footnotetext{
${ }^{22}$ Courant, Fredrichs, and Lewy, Math. Ann. 100, 32 (1928).
}

Pasadena, California) in the computing center at the California Institute of Technology. Floating point numbers were used. The total time required was about 35 hours.

\section{RESULTS AND DISCUSSION}

The calculation yields the dimensionless functions $u(\rho, \tau), q_{A}(\tau)$, and $-d q_{A}(\tau) / d \tau$. The first is readily converted to $\gamma(\rho, \tau)$, which gives the radial concentration profiles of the species $A$. In tabulating and plotting the results, we have followed general practice and given the fractional attainment of equilibrium, $F(\tau)$, rather than $q_{A}(\tau)$. Under the initial and boundary conditions (12), this quantity is given by

$$
F(\tau)=1-q_{A}(\tau) .
$$

The representation of the data is kept in dimensionless form, since the actual values of the concentrations, the fractional attainment of equilibrium, and the rates at a given time $t$ depend also on $D_{A}, r_{0}, C$, and $V$. They can be obtained from the tables and graphs by substituting numerical values for these quantities and using (6) and (9).

Table I gives the fractional attainment of equilibrium, $F(\tau)$, and the dimensionless rate, $-d q_{A} / d \tau$, for the various cases. Figure 2 shows a plot of $F$ versus $\tau$ and includes the solution to the linear case $\left(D_{A}=D_{B}\right)$ which is well known ${ }^{4}$ to be

$$
F(\tau)=1-\frac{6}{\pi^{2}} \sum_{n=1}^{\infty} \frac{1}{n^{2}} \exp \left(-n^{2} \pi^{2} \tau\right) .
$$

It should be emphasized that the time coordinate $\tau$ contains the quantity $D_{A}$; hence, these results can be used for direct comparison of different processes onlv if the ion $A$ initially present in the beads is the same in all cases. Figure 2 shows that the curves for different ratios $D_{A} / D_{B}$ cannot be transformed into one another by a linear transformation of the time. If the ion $B$ is slower, the process is slower, but not by a constant factor, because the interdiffusion coefficient increases with decreasing concentration of $A$. On the other hand, if $B$ is faster, the process is faster, but the interdiffusion coefficient decreases during the process.

It is of interest to compare the rates for forward and backward exchange of two counter ion species. In Fig. 3 we have plotted the fractional attainment of equilibrium for the exchange processes $\mathrm{H}^{+}$against $\mathrm{Li}^{+}$and $\mathrm{Li}^{+}$ against $\mathrm{H}^{+}$, assuming a ratio $D_{\mathrm{H}} / D_{\mathrm{Li}}=10$. For direct comparison we have chosen $D_{\mathrm{H}} t / r_{0}{ }^{2}$ as the dimensionless time coordinate in both cases. It is seen that the process is faster if $\mathrm{H}^{+}$, the faster ion, is initially present in the resin. The half-times of exchange differ by about the factor 2 , the time required for $90 \%$ exchange by about the factor 3. According to previous theories, which assume a constant interdiffusion coefficient, the two curves should coincide.

The radial concentration profiles of the species $A$ for 
TABLE I. Fractional attainment of equilibrium, $F(\tau)$, and dimensionless rate, $-d q_{A}(\tau) / d \tau$, for six different values of the ratio $D_{A} / D_{B}$.

\begin{tabular}{|c|c|c|c|c|c|c|c|c|c|c|c|}
\hline & & $d q_{A}(r)$ & & & & & & $d A A(\tau)$ & & & $d Q_{A}(\tau)$ \\
\hline $\boldsymbol{x}$ & $F(\tau)$ & $d r$ & $T$ & $F(\tau)$ & $d \tau$ & $\tau$ & $F(x)$ & $d \tau$ & $\tau$ & $F(t)$ & $d r$ \\
\hline \multicolumn{6}{|c|}{ (a) $D_{A} / D_{B}=10$} & \multicolumn{6}{|c|}{ (c) $D_{A} / D_{B}=2$} \\
\hline 0.00001831 & 0.006 & & 0.08187 & 0.477 & 2.52 & 0.0003113 & 0.051 & & 0.06031 & 0.591 & 3.81 \\
\hline 0.00007690 & 0.012 & & 0.08937 & 0.495 & 2.37 & 0.001249 & 0.101 & & 0.06406 & 0.605 & 3.63 \\
\hline 0.0003113 & 0.023 & & 0.09687 & 0.512 & 2.24 & 0.001718 & 0.118 & 33.55 & 0.06781 & 0.618 & 3.47 \\
\hline 0.001717 & 0.051 & 27.60 & 0.1044 & 0.528 & 2.12 & 0.002186 & 0.133 & 29.52 & 0.07156 & 0.631 & 3,32 \\
\hline 0.002185 & 0.063 & 25.33 & 0.1119 & 0.544 & 2.02 & 0.002655 & 0.146 & 26.61 & 0.07531 & 0.643 & 3.18 \\
\hline 0.002654 & 0.074 & 23.10 & 0.1231 & 0.566 & 1.88 & 0.003124 & 0.157 & 24.36 & 0.07906 & 0.655 & 3.05 \\
\hline 0.003122 & 0.084 & 20.97 & 0.1306 & 0.580 & 1.80 & 0.003593 & 0.168 & 22.56 & 0.08281 & 0.666 & 2.92 \\
\hline 0.003592 & 0.093 & 19.02 & 0.1456 & 0.606 & 1.65 & 0.004062 & 0.178 & 21.07 & 0.08656 & 0.677 & 2.81 \\
\hline 0.004060 & 0.102 & 17.31 & 0.1606 & 0.629 & 1.53 & 0.004530 & 0.188 & 19.30 & 0.09031 & 0.687 & 2.70 \\
\hline 0.004529 & 0.110 & 15.88 & 0.1756 & 0.652 & 1.42 & 0.004999 & 0.197 & 18.76 & 0.09406 & 0.697 & 2.61 \\
\hline 0.004998 & 0.117 & 14.72 & 0.1906 & 0.672 & 1.33 & 0.005963 & 0.214 & 17.03 & 0.1016 & 0.716 & 2.43 \\
\hline 0.005935 & 0.130 & 13.02 & 0.2056 & 0.691 & 1.24 & 0.006874 & 0.229 & 15.66 & 0.1091 & 0.733 & 2.26 \\
\hline 0.006873 & 0.141 & 11.84 & 0.2206 & 0.709 & 1.16 & 0.007811 & 0.243 & 14.55 & 0.1166 & 0.750 & 2.12 \\
\hline 0.007810 & 0.152 & 10.94 & 0.2356 & 0.726 & 1.11 & 0.008749 & 0.256 & 13.62 & 0.1241 & 0.765 & 1.98 \\
\hline 0.008748 & 0.161 & 10.20 & 0.2506 & 0.742 & 1.03 & 0.009686 & 0.268 & 12.83 & 0.1316 & 0.779 & 1.86 \\
\hline 0.01062 & 0.179 & 9.04 & 0.2656 & 0.757 & 0.976 & 0.01062 & 0.280 & 12.15 & 0.1391 & 0.793 & 1.74 \\
\hline 0.01437 & 0.213 & 7.37 & 0.2806 & 0.771 & 0.923 & 0.01203 & 0.296 & 11.28 & 0.1466 & 0.805 & 1.64 \\
\hline 0.01812 & 0.237 & 6.42 & 0.2956 & 0.785 & 0.874 & 0.01344 & 0.312 & 10.55 & 0.1541 & 0.817 & 1.54 \\
\hline 0.02187 & 0.259 & 5.77 & 0.3106 & 0.798 & 0.829 & 0.01484 & 0.326 & 9.93 & 0.1616 & 0.828 & 1.45 \\
\hline 0.02562 & 0.280 & 5.27 & 0.3331 & 0.815 & 0.766 & 0.01625 & 0.340 & 9.42 & 0.1691 & 0.839 & 1.37 \\
\hline 0.02937 & 0.299 & 4.87 & 0.3556 & 0.832 & 0.710 & 0.01812 & 0.357 & 8.80 & 0.1841 & 0.858 & 1.21 \\
\hline 0.03312 & 0.317 & 4.53 & 0.3781 & 0.847 & 0.658 & 0.02000 & 0.373 & 8.27 & 0.1991 & 0.875 & 1.07 \\
\hline 0.03687 & 0.333 & 4.24 & 0.3856 & 0.852 & 0.642 & 0.02187 & 0.388 & 7.80 & 0.2141 & 0.890 & 0.957 \\
\hline 0.04062 & 0.348 & 3.99 & 0.4156 & 0.870 & 0.582 & 0.02375 & 0.402 & 7.40 & 0.2291 & 0.904 & 0.848 \\
\hline 0.04437 & 0.363 & 3.77 & 0.4456 & 0.887 & 0.527 & 0.02656 & 0.422 & 6.87 & 0.2441 & 0.916 & 0.753 \\
\hline 0.04812 & 0.376 & 3.58 & 0.4756 & 0.902 & 0.476 & 0.03031 & 0.446 & 6.29 & 0.2591 & 0.926 & 0.666 \\
\hline 0.05187 & 0.389 & 3.41 & 0.5056 & 0.915 & 0.430 & 0.03406 & 0.469 & 5.81 & 0.2891 & 0.944 & 0.518 \\
\hline 0.05562 & 0.402 & 3,26 & 0.5656 & 0.939 & 0.346 & 0.03781 & 0.490 & 5.40 & 0.3191 & 0.958 & 0.399 \\
\hline 0.05937 & 0.414 & 3.12 & 0.6256 & 0.957 & 0.273 & 0.04156 & 0.509 & 5.05 & 0.3641 & 0.972 & 0.266 \\
\hline 0.06312 & 0.425 & 3.00 & 0.6856 & 0.971 & 0.206 & 0.04531 & 0.528 & 4.74 & 0.4091 & 0.982 & 0.175 \\
\hline 0.06687 & 0.436 & 2.89 & 0.7456 & 0.982 & 0.147 & 0.04906 & 0.545 & 4.47 & 0.4691 & 0.990 & 0.099 \\
\hline
\end{tabular}

0.07437

(b) $D_{A} / D_{B}=5$

0.00001831
0.00007690
0.0003113
0.001718
0.002186
0.002655
0.003124
0.003593
0.004061
0.004999
0.005963
0.006874
0.007811
0.008750
0.009686
0.01062
0.01156
0.01297
0.01437
0.01578
0.01766
0.01953
0.02141
0.02328
0.02516
0.02844
0.03219
0.03969
0.04719
0.05469

0.010

0.020

0.041

0.095

0.117

0.127

0.135

0.144

0.172

0.185

0.197

0.208

0.218

0.228

0.237

0.250

0.262

0.274

0.289

0.302

0.315

0.328

0.342

0.358

0.379

0.414

0.448
0.477

27.11
23.37
20.97
19.35
18.13
17.12
15.43
14.06
12.96
12.06
11.31
10.67
10.13
9.65
9.04
8.51
8.06
7.55
7.11
6.73
6.39
6.03
.5 .66
5.24
4.58
4.10
3.70

0.8356

0.991

0.075

$\begin{array}{lll}0.06219 & 0.503 & 3.39 \\ 0.06969 & 0.527 & 3.12 \\ 0.07719 & 0.550 & 2.90 \\ 0.08469 & 0.571 & 2.70 \\ 0.09219 & 0.590 & 2.53 \\ 0.09969 & 0.609 & 2.38 \\ 0.1072 & 0.626 & 2.25 \\ 0.1147 & 0.644 & 2.13 \\ 0.1222 & 0.658 & 2.02 \\ 0.1297 & 0.673 & 1.91 \\ 0.1372 & 0.687 & 1.82 \\ 0.1447 & 0.700 & 1.74 \\ 0.1597 & 0.725 & 1.59 \\ 0.1747 & 0.748 & 1.45 \\ 0.1897 & 0.769 & 1.34 \\ 0.2047 & 0.788 & 1.23 \\ 0.2197 & 0.806 & 1.14 \\ 0.2347 & 0.822 & 1.05 \\ 0.2497 & 0.837 & 0.977 \\ 0.2647 & 0.851 & 0.906 \\ 0.2872 & 0.870 & 0.810 \\ 0.3097 & 0.888 & 0.724 \\ 0.3322 & 0.903 & 0.645 \\ 0.3622 & 0.921 & 0.555 \\ 0.3922 & 0.936 & 0.471 \\ 0.4222 & 0.949 & 0.395 \\ 0.4522 & 0.960 & 0.328 \\ 0.5122 & 0.976 & 0.215 \\ 0.5722 & 0.986 & 0.133 \\ 0.6622 & 0.994 & 0.059\end{array}$

(c) $D_{A} / D_{B}=2$

$0.00001831 \quad 0.013$

0.00007690

$0.05281 \quad 0.561 \quad 4.23$

$\begin{array}{lll}0.05656 & 0.576 & 4.01\end{array}$ (d) $D_{A} / D_{B}=\frac{1}{2}$

\begin{tabular}{llllll}
0.00001221 & 0.014 & & 0.03002 & 0.539 & 7.07 \\
0.00005127 & 0.027 & & 0.03252 & 0.556 & 6.64 \\
0.0001802 & 0.049 & & 0.03502 & 0.572 & 6.25 \\
0.0006489 & 0.092 & & 0.03752 & 0.587 & 5.91 \\
0.0009614 & 0.113 & 58.73 & 0.04002 & 0.601 & 5.60 \\
0.001274 & 0.130 & 50.17 & 0.04252 & 0.615 & 5.31 \\
0.001586 & 0.145 & 44.36 & 0.04502 & 0.628 & 5.05 \\
0.001899 & 0.157 & 40.08 & 0.04752 & 0.640 & 4.82 \\
0.002211 & 0.169 & 36.78 & 0.05002 & 0.652 & 4.60 \\
0.002524 & 0.180 & 34.12 & 0.05252 & 0.663 & 4.39 \\
0.002836 & 0.190 & 31.92 & 0.05502 & 0.674 & 4.20 \\
0.003149 & 0.200 & 30.07 & 0.05752 & 0.684 & 4.03 \\
0.003774 & 0.218 & 27.08 & 0.06002 & 0.694 & 3.86 \\
0.004399 & 0.234 & 24.77 & 0.06252 & 0.703 & 3.71 \\
0.005024 & 0.248 & 22.91 & 0.06752 & 0.721 & 3.43 \\
0.005649 & 0.262 & 21.37 & 0.07252 & 0.738 & 3.17 \\
0.006274 & 0.275 & 20.07 & 0.07752 & 0.753 & 2.95 \\
0.006899 & 0.288 & 18.95 & 0.08252 & 0.767 & 2.75 \\
0.007524 & 0.299 & 17.97 & 0.08752 & 0.780 & 2.57 \\
0.008774 & 0.320 & 16.31 & 0.09252 & 0.792 & 2.40 \\
0.01002 & 0.340 & 15.03 & 0.09752 & 0.804 & 2.24 \\
0.01127 & 0.358 & 13.95 & 0.1025 & 0.815 & 2.10 \\
0.01252 & 0.375 & 13.04 & 0.1075 & 0.825 & 1.97 \\
0.01377 & 0.390 & 12.26 & 0.1125 & 0.835 & 1.85 \\
0.01502 & 0.405 & 11.57 & 0.1225 & 0.852 & 1.63 \\
0.01627 & 0.419 & 10.96 & 0.1325 & 0.867 & 1.45 \\
0.01752 & 0.433 & 10.42 & 0.1475 & 0.887 & 1.21 \\
0.01877 & 0.445 & 9.94 & 0.1625 & 0.904 & 1.02 \\
0.02002 & 0.457 & 9.50 & 0.1825 & 0.922 & 0.827 \\
0.02127 & 0.469 & 9.10 & 0.2025 & 0.937 & 0.664 \\
0.02252 & 0.480 & 8.73 & 0.2225 & 0.949 & 0.535 \\
0.02377 & 0.491 & 8.40 & 0.2625 & 0.966 & 0.351 \\
0.02502 & 0.501 & 8.08 & 0.3225 & 0.981 & 0.189 \\
0.02752 & 0.520 & 7.53 & 0.4025 & 0.992 & 0.084 \\
& & & & & \\
\hline & & & & &
\end{tabular}


TABLE I.-Continued.

\begin{tabular}{|c|c|c|c|c|c|c|c|c|c|c|c|}
\hline & & $-d q_{A}(\tau)$ & & & $d q_{A}(\tau)$ & & & $d q_{A}(\tau)$ & & & $d q_{A}(\tau)$ \\
\hline$\tau$ & $F(\tau)$ & $d \tau$ & $r$ & $F(\tau)$ & $d \tau$ & $\tau$ & $F(\tau)$ & $d \tau$ & $\tau$ & $F(\tau)$ & $d \tau$ \\
\hline \multicolumn{6}{|c|}{ (e) $D_{A} / D_{B}=\frac{1}{5}$} & \multicolumn{6}{|c|}{ (f) $D_{A} / D_{B}=\frac{1}{10}$} \\
\hline 0.000006104 & 0.011 & & 0.02532 & 0.543 & 8.18 & 0.000004272 & 0.010 & & 0.02408 & 0.551 & 8.47 \\
\hline 0.00002564 & 0.023 & & 0.02782 & 0.562 & 7.58 & 0.00001794 & 0.019 & & 0.02583 & 0.565 & 7.99 \\
\hline 0.00008423 & 0.039 & & 0.03032 & 0.580 & 7.05 & 0.00007263 & 0.037 & & 0.02758 & 0.579 & 7.56 \\
\hline 0.0004749 & 0.090 & 93.11 & 0.03282 & 0.597 & 6.95 & 0.0003461 & 0.078 & 115.6 & 0.02933 & 0.592 & 7.16 \\
\hline 0.0006311 & 0.103 & 79.96 & 0.03532 & 0.613 & 6.18 & 0.0004554 & 0.090 & 99.31 & 0.03108 & 0.604 & 6.80 \\
\hline 0.0007874 & 0.115 & 70.94 & 0.03782 & 0.628 & 5.81 & 0.0005648 & 0.101 & 88.32 & 0.03283 & 0.616 & 6.48 \\
\hline 0.0009436 & 0.125 & 64.26 & 0.04032 & 0.642 & 5.47 & 0.0006742 & 0.111 & 80.22 & 0.03458 & 0.627 & 6.17 \\
\hline 0.001100 & 0.135 & 59.07 & 0.04282 & 0.656 & 5.17 & 0.0007836 & 0.119 & 73.92 & 0.03720 & 0.643 & 5.78 \\
\hline 0.001412 & 0.152 & 51.42 & 0.04532 & 0.668 & 4.89 & 0.001002 & 0.134 & 64.61 & 0.03983 & 0.658 & 5.41 \\
\hline 0.001725 & 0.167 & 45.98 & 0.04782 & 0.680 & 4.66 & 0.001221 & 0.148 & 57.94 & 0.04245 & 0.672 & 5.08 \\
\hline 0.002037 & 0.181 & 41.85 & 0.05032 & 0.692 & 4.42 & 0.001440 & 0.160 & 52.87 & 0.04508 & 0.684 & 4.79 \\
\hline 0.002506 & 0.199 & 37.18 & 0.05282 & 0.702 & 4.21 & 0.001659 & 0.171 & 48.84 & 0.04770 & 0.696 & 4.52 \\
\hline 0.002975 & 0.216 & 33.68 & 0.05782 & 0.722 & 3.82 & 0.001987 & 0.186 & 44.09 & 0.05033 & 0.708 & 4.27 \\
\hline 0.003444 & 0.231 & 30.92 & 0.06282 & 0.740 & 3.49 & 0.002315 & 0.200 & 40.39 & 0.05558 & 0.729 & 3.84 \\
\hline 0.004069 & 0.249 & 28.02 & 0.06782 & 0.757 & 3.19 & 0.002643 & 0.213 & 37.41 & 0.06258 & 0.754 & 3.36 \\
\hline 0.004694 & 0.266 & 25.72 & 0.07282 & 0.772 & 2.94 & 0.003080 & 0.230 & 34.21 & 0.06958 & 0.776 & 2.96 \\
\hline 0.005318 & 0.281 & 23.84 & 0.07782 & 0.786 & 2.71 & 0.003955 & 0.258 & 28.87 & 0.07658 & 0.796 & 2.63 \\
\hline 0.005944 & 0.296 & 22.27 & 0.08282 & 0.799 & 2.50 & 0.004830 & 0.282 & 25.68 & 0.08533 & 0.818 & 2.29 \\
\hline 0.006569 & 0.309 & 20.93 & 0.08782 & 0.811 & 2.32 & 0.005705 & 0.304 & 23.22 & 0.09408 & 0.836 & 2.00 \\
\hline 0.007819 & 0.334 & 18.80 & 0.09532 & 0.828 & 2.08 & 0.006580 & 0.324 & 21.26 & 0.1028 & 0.853 & 1.76 \\
\hline 0.009069 & 0.356 & 17.10 & 0.1028 & 0.843 & 1.87 & 0.007455 & 0.342 & 19.64 & 0.1116 & 0.867 & 1.56 \\
\hline 0.01032 & 0.376 & 15.71 & 0.1103 & 0.856 & 1.68 & 0.008330 & 0.359 & 18.28 & 0.1203 & 0.880 & 1.38 \\
\hline 0.01157 & 0.395 & 14.55 & 0.1178 & 0.868 & 1.52 & 0.009205 & 0.375 & 17.12 & 0.1291 & 0.892 & 1.23 \\
\hline 0.01282 & 0.413 & 13.56 & 0.1253 & 0.879 & 1.38 & 0.01008 & 0.391 & 16.11 & 0.1378 & 0.902 & 1.10 \\
\hline 0.01407 & 0.429 & 12.71 & 0.1398 & 0.889 & 1.25 & 0.01096 & 0.404 & 15.22 & 0.1553 & 0.919 & 0.90 \\
\hline 0.01532 & 0.444 & 11.97 & 0.1441 & 0.902 & 1.09 & 0.01183 & 0.416 & 14.44 & 0.1728 & 0.933 & 0.73 \\
\hline 0.01657 & 0.459 & 11.31 & 0.1591 & 0.917 & 0.917 & 0.01358 & 0.440 & 13.10 & 0.1903 & 0.945 & 0.55 \\
\hline 0.01782 & 0.473 & 10.72 & 0.1741 & 0.929 & 0.769 & 0.01533 & 0.463 & 11.99 & 0.2078 & 0.954 & 0.49 \\
\hline 0.01907 & 0.486 & 10.20 & 0.1891 & 0.940 & 0.649 & 0.01708 & 0.483 & 11.07 & 0.2253 & 0.962 & 0.40 \\
\hline 0.02032 & 0.498 & 9.72 & 0.2191 & 0.956 & 0.463 & 0.01883 & 0.502 & 10.27 & 0.2603 & 0.973 & 0.27 \\
\hline 0.02159 & 0.510 & 9.29 & 0.2641 & 0.972 & 0.286 & $\begin{array}{l}0.01883 \\
0.02058\end{array}$ & 0.519 & 9.58 & 0.2953 & 0.981 & 0.19 \\
\hline 0.02282 & $\begin{array}{l}0.521 \\
0.532\end{array}$ & $\begin{array}{l}8.89 \\
8.52\end{array}$ & $\begin{array}{l}0.3091 \\
0.3691\end{array}$ & $\begin{array}{l}0.982 \\
0.994\end{array}$ & $\begin{array}{l}0.179 \\
0.097\end{array}$ & $\begin{array}{l}0.02058 \\
0.02233\end{array}$ & 0.535 & 9.01 & 0.3378 & 0.991 & 0.11 \\
\hline 0.0240 & & & & & & & & & & & \\
\hline
\end{tabular}

$25 \%, 50 \%, 75 \%$, and $90 \%$ exchange are shown in Fig. 4. The profiles for the linear case $\left(D_{A}=D_{B}\right)$ given by $^{4}$

$$
\gamma(\rho, \tau)=-\frac{2}{\pi \rho} \sum_{n=1}^{\infty} \frac{(-1)^{n}}{n} \sin (n \pi \rho) \exp \left(-n^{2} \pi^{2} \tau\right)
$$

are included. The shape of the profiles depends strongly on the ratio $D_{A} / D_{B}$. If the ion present in the sphere initially is much the faster one, a comparatively sharp boundary moves in toward the center of the bead [Fig. 4(a)]. If the ion present in the bead initially is much the slower one, the boundary is diffuse and the process reaches the center rapidly [Fig. 4(b)]. Again, the explanation is straightforward. In the first case the concentration of the faster ion is small near the surface and large near the center of the bead. Hence, the interdiffusion coefficient decreases toward the center. Therefore the outer shells of the bead are exhausted
Fig. 2. Fractional attainment of equilibrium as a function of the dimensionless time coordinate $\tau=D_{A} t / r_{0}^{2}$. The different curves correspond to different ratios $D_{A} / D_{B}$. The solution to the linear problem $\left(D_{A}=D_{B}\right)$ calculated from Eq. (19) is given as a broken line.

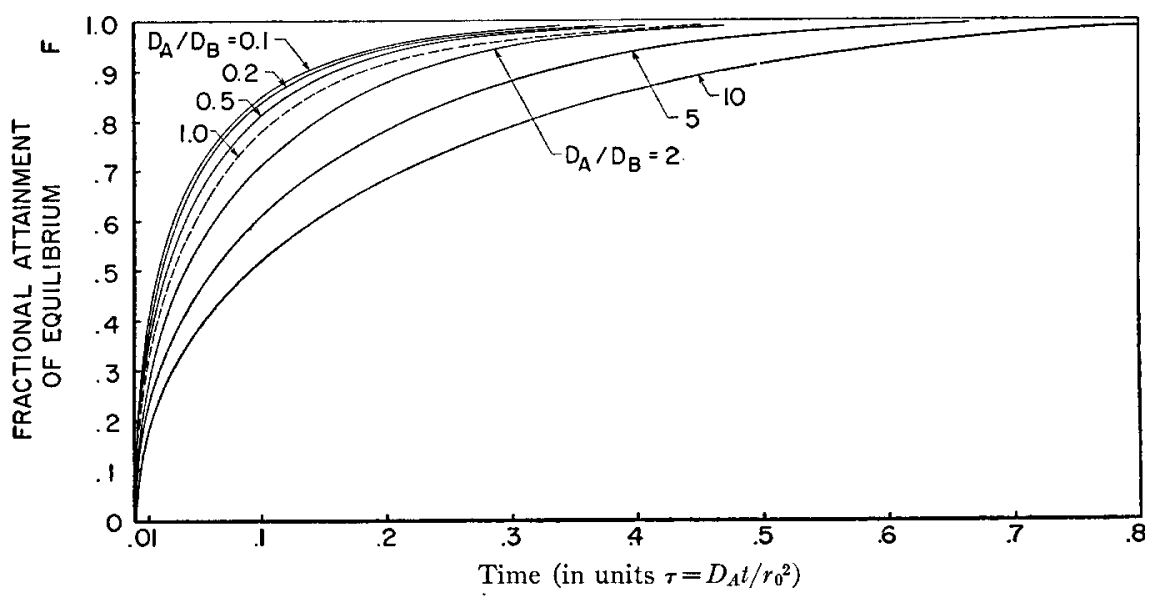




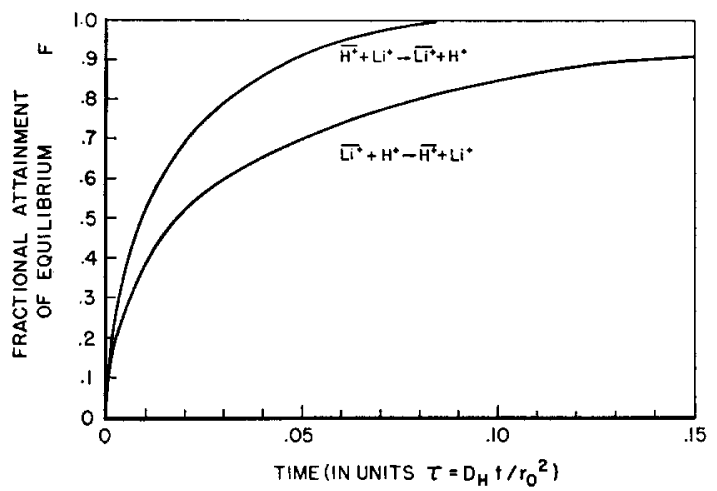

FIG. 3. Fractional attainment of equilibrium for the exchange processes $\mathrm{H}^{+}$(resin) $+\mathrm{Li}^{+}$(solution) $\rightarrow \mathrm{Li}^{+}$(resin) $+\mathrm{H}^{+}$(solution), and $\mathrm{Li}^{+}$(resin) $+\mathrm{H}^{+}$(solution) $\rightarrow \mathrm{H}^{+}$(resin) $+\mathrm{Li}^{+}$(solution, assuming a ratio $D_{\mathrm{H}} / D_{\mathrm{Li}}=10$. In the first case (upper curve) the exchange is faster.

rapidly, whereas the exchange near the center remains slow. In the second case, the opposite holds. The interdiffusion coefficient increases toward the center; therefore the exhaustion is more uniform. Previous theories lead to one set of curves for all cases [Fig. 4(g)].

For practical purposes, an explicit expression for $F(\tau)$ which approximates the numerical results will be useful. The relation

$$
F(\tau)=\left(1-\exp \left\{\pi^{2}\left[f_{1}(\alpha) \tau+f_{2}(\alpha) \tau^{2}+f_{3}(\alpha) \tau^{3}\right]\right\}\right)^{\frac{1}{2}},
$$

where $\alpha \equiv D_{A} / D_{B}$ and the coefficients $f_{1}(\alpha), f_{2}(\alpha)$, and $f_{3}(\alpha)$ are given by

$$
\begin{aligned}
& 1 / f_{1}(\alpha)=-0.570-0.430 \alpha^{0.775} \\
& 1 / f_{2}(\alpha)=0.260+7.82 \alpha \\
& 1 / f_{3}(\alpha)=-0.165-0.177 \alpha
\end{aligned}
$$

fits all the numerical results within an error of \pm 0.01 in $F(\tau)$, with the exception of $\alpha=0.1$, where the maximum error is -0.02 . It should hold equally well for intermediate values of the ratio $D_{A} / D_{B}$. Equation (21) was developed as an extension of a simpler but less accurate approximation given by Vermeulen ${ }^{23}$ for the linear case.

We wish to emphasize again that the results represent ideal limiting laws only, from which the behavior of actual systems may be expected to show more or less pronounced deviations. However, the comparison with the solution for the linear case demonstrates conclusively that the effect of the electric potential included in our approach is an essential feature of the process which no theory can omit.

\section{EXTENSION TO OTHER INITIAL DISTRIBUTIONS}

The numerical results and the empirical formula given above may be used to determine the solution for

\footnotetext{
${ }^{23}$ T. Vermeulen, Ind. Eng. Chem. 45, 1664 (1953).
}
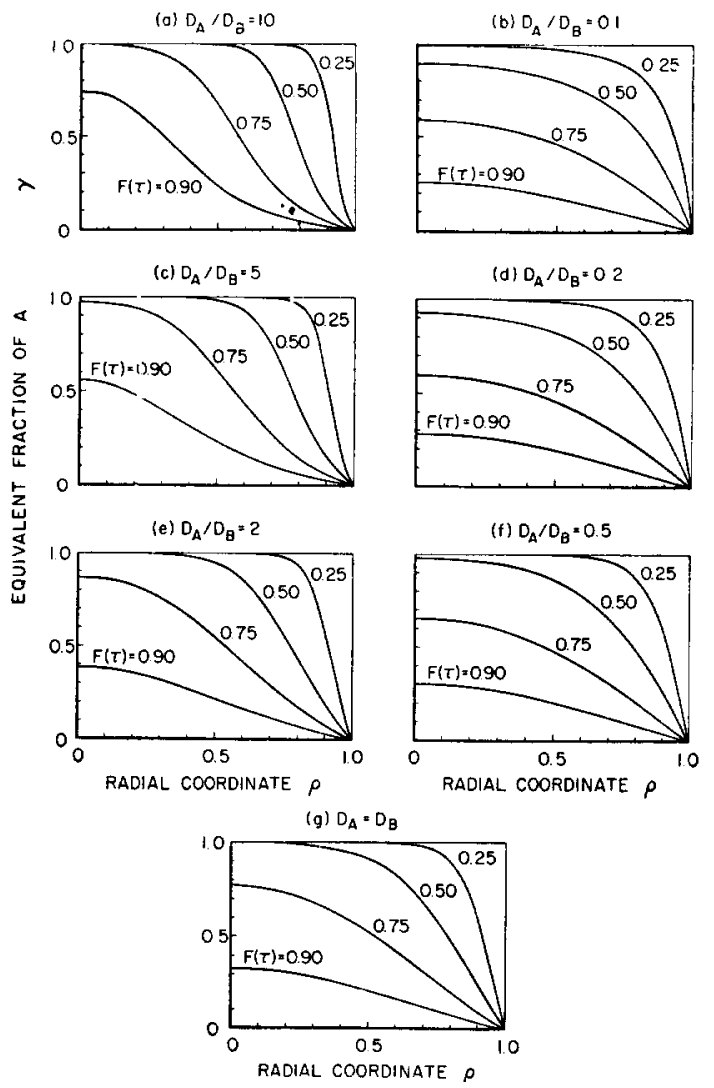

Fig. 4. Concentration profiles of the species $A$ in the bead for $25 \%, 50 \%, 75 \%$, and $90 \%$ exchange $(F=0.25,0.50,0.75$, and $0.90)$ for seven different ratios $D_{A} / D_{B}$. The profiles for the linear case $\left(D_{A}=D_{B}\right)$ were calculated from Eq. (20).

the problem in which the distribution is

$$
\begin{array}{rlrl}
0 \leq \rho<1, & \tau=0, & \gamma(\rho) & =\gamma_{0} \\
\rho \geq 1, & \gamma(\rho, \tau) & =0
\end{array}
$$

where $\gamma_{0}$ may have any constant value in the range $0<\gamma_{0} \leq 1$. The procedure is as follows. One replaces $\gamma$ by $\gamma^{\prime}=\gamma / \gamma_{0}$ and $a$ by $a^{\prime}=a \gamma_{0}$. Then $\gamma^{\prime}$ satisfies the same differential equation and the same boundary values as in the problem already described. The solution for the new problem as presented in (21) and in the numerical tables is now in terms of $a^{\prime}$ and $\gamma^{\prime}$. In this way, the solution is available for any uniform initial value of $\gamma$ in the sphere.

\section{ACKNOWLEDGMENTS}

We are indebted to Dr. J. Franklin, Dr. C. B. Ray, and Dr. R. Nathan for helpful suggestions concerning the numerical integration. We also wish to express our gratitude to Mr. D. Brouillette and Miss Z. Lindberg for their valuable assistance in the computations. 